\title{
Telomere biology of Baikal hydrobionts
}

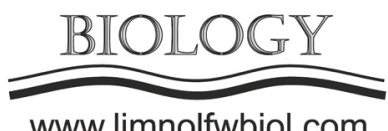

www.limnolfwbiol.com

\author{
Koroleva A.G. ${ }^{1 *}$, Maximova N.V. ${ }^{1}$, Sapozhnikova Yu.P. ${ }^{1}$, Timoshkin O.A. ${ }^{1}$, \\ Zaytseva E.P. ${ }^{1}$, Sitnikova T.Ya. ${ }^{1}$, Evtushenko E.V.2, Vershinin A.V.2, \\ Sukhanova L.V.1 ${ }^{1}$ Glyzina O.Yu' ${ }^{1}$, Kirilchik S.V. ${ }^{1}$
}

\begin{abstract}
${ }^{1}$ Limnological Institute, Siberian Branch of the Russian Academy of Sciences, Ulan-Batorskaya Str., 3, Irkutsk, 664033, Russia
${ }^{2}$ Institute of Molecular and Cellular Biology, Siberian Branch of the Russian Academy of Sciences, Acad. Lavrentiev Ave., 8/2, Novosibirsk, 630090 Russia
\end{abstract}

\begin{abstract}
Telomeres are the end regions of eukaryotic chromosomes. In addition to maintaining the integrity of the genome, they are involved in aging, regeneration, stress, and disease. We study the telomere biology in Baikal and Siberian planarians, mollusks, and fish. Using these hydrobionts as an example, we study how changes in body size, life expectancy, reproductive strategy, and habitat have reflected in telomeres during evolution, how telomere length changes during ontogeny, and whether stress factors affect its dynamics. We observe both the common features in the telomere biology of such different hydrobionts and their species-specific features. The obtained data indicate a possible influence of physiological and environmental features on the functioning of telomeres.
\end{abstract}

Keywords: telomere biology, Lake Baikal hydrobionts, mollusks, planarians, fish

Particular interest in telomeres, the terminal regions of chromosomes, arose in the early 90 s of the 20th century, when it became known that telomeres shorten during aging (Harley et al., 1990). Since then, much has become known about the structure and functions of telomeres. Data appeared not only on the detailed structure of telomeres in different organisms, the evolution of telomeric repeats (Fulneckova et al., 2013), and their important role in cell functioning, which is not limited to maintaining the integrity of the genome (Zhu et al., 2019), but also on the sensitivity of telomeres to various external factors of ecological (Beaulieu et al., 2017), social (Lewin et al., 2015), and psycho-emotional nature (Simon et al., 2006).

The special conditions for the formation of Lake Baikal contributed to the occurrence of a diverse and specific fauna (Matz et al., 2011). Each group of hydrobionts is rich in endemic species, the number of which in some taxa may be close to $100 \%$, as, for example, in the order of flatworms, Tricladida (Timoshkin, 1994). Due to the unique ecological situation, the study of telomeres in Baikal hydrobionts can make a significant contribution to understanding how the organism interacts with the environment at the molecular level and what specific features in telomere biology appeared in organisms during evolution.

We study the structure, length, age dynamics of telomere length (TL), and the activity of telomerase, the enzyme that regulates TL, in Baikal and Siberian planarians, mollusks, and fish. Using these hydrobionts as an example, we study how changes in body size, life expectancy, reproductive strategy, and habitat have reflected in telomeres during evolution as well as how TL changes during ontogeny, and whether stress factors affect its dynamics.

In planarians, mollusks, and fish, telomere DNA consists of TTAGGG repeats. What unites these hydrobionts is the fact that newborns have longer telomeres than adults. However, the TL, its dynamics, and telomerase activity are, as a rule, species-specific and may differ in sister species. The studied planarian species (Rimacephalus arecepta, Sorocelis hepatizon, Baikalobia guttata, and Phagocata sibirica) revealed three types of age-related telomere dynamics: shortening at the early stages of ontogeny and subsequent maintenance, gradual shortening and cyclic dynamics (Koroleva et al., 2020). In mollusks (Benedictia fragilis, B. baicalensis, and Kobeltocochlea martensiana), sexual specificity was observed in the telomere dynamics during aging: in females, maintenance or increase in $\mathrm{TL}$, in males, increase, maintenance and shortening of TL during ontogeny, which could be associated with environmental conditions and different reproductive strategies of these species (Maximova et al., 2017). In the peled Coregonus peled, we observed a TL shortening in the first two years of life (Koroleva et al., 2019). The

*Corresponding author.

E-mail address: ankor-2015@yandex.ru (A.G. Koroleva)

(C) Author(s) 2020. This work is distributed under the Creative Commons Attribution 4.0 License. 
response to stress can also be different and lead either to an increase in TL (preliminary data, in the ears of the stone sculpin Paracottus knerii) or TL shortening (in the brain of $P$. knerii and $C$. peled). In dioecious mollusks and fish, there may be differences in TL between sexes in some species (females have longer telomeres than males in the $B$. fragilis and $B$. baicalensis mollusks as well as the $P$. knerii fish); in other species, there may be no differences (the $K$. martensiana and the $C$. peled fish).

Thus, we observe the common features in the telomere biology of such different hydrobionts as planarians, mollusks, and fish as well as the features characteristic for individual species. The universality of the telomeric repeat indicates its importance, although changes have repeatedly occurred in the structure of telomeres in different groups of eukaryotes (Georgiev et al., 2000). Another common feature also indicates that the rapid cell division accompanying the growth of a body leads to the loss of telomeric repeats in different organisms (Frenck et al., 1998; Salomons et al., 2009). At the same time, numerous differences in the telomere biology between species point to the possible influence of physiological and environmental features on the functioning of telomeres.

This study was carried out at the LIN SB RAS Collective Instrumental Center (http://www.lin.irk. ru/copp/eng/) using the unique scientific installation Experimental Freshwater Aquarium Complex for Baikal Hydrobionts at LIN SB RAS supported by RFBR and the Government of the Irkutsk Region, projects Nos. 17-44388081 r_a and 17-44-388106 r_a, RFBR projects Nos. 12-04-32052 and 13-04-01270, and SB RAS integration projects, Nos. 45, 37, and 51, within the framework of the State Tasks No. 0345-2019-0002 (AAAA-A16116122110066-1) "Molecular Ecology and Evolution of Living Systems ..." and No. 0345-2019-0009 (AAAAA16-116122110067-8) "Large-Scale Changes in the Ecology and Biodiversity of Lake Baikal ...”.

\section{References}

Beaulieu M., Benoit L., Abaga S. et al. 2017. Mind the cell: seasonal variation in telomere length mirrors changes in leucocyte profile. Molecular Ecology 26: 5603-5613. DOI: 10.1111/mec.14329
Frenck R.W.Jr., Blackburn E.H., Shannon K.M. 1998. The rate of telomere sequence loss in human leukocytes varies with age. PNAS 95: 5607-5610. DOI: 10.1073/pnas.95.10.5607

Fulneckova J., Sevcikova T., Fajkus J. et al. 2013. A broad phylogenetic survey unveils the diversity and evolution of telomeres in eukaryotes. Genome Biology and Evolution 5: 468-483. DOI: 10.1093/gbe/evt019

Georgiev P.G., Melnikova L.S., Kan T.G. et al. 2000. Different mechanisms of telomere length regulation. Molecular Biology 34: 628-636. DOI: 10.1007/BF02759599

Harley C.B., Futcher A.B., Greider C.W. 1990. Telomeres shorten during ageing of human fibroblasts. Nature 345: 458-460. DOI: $10.1038 / 345458 \mathrm{a} 0$

Koroleva A.G., Evtushenko E.V., Vershinin A.V. et al. 2020. Age dynamics of telomere length in endemic Baikal planarians. Molecular Biology (Russia) 54. DOI: 10.31857/ S0026898420040072 (in press)

Koroleva A.G., Sapozhnikova Yu.P., Tyagun M.L. et al. 2019. Telomere length decreases during early life stages in peled. Limnology and Freshwater Biology 6: 326-331. DOI:10.31951/2658-3518-2019-A-6-326

Lewin N., Treidel L.A., Holekamp K.E. et al. 2015. Socioecological variables predict telomere length in wild spotted hyenas. Biology Letters 11. DOI: 10.1098/ rsbl.2014.0991

Matz V.D., Scherbakov D.Yu., Efimova I.M. 2011. Late Cretaceous-Cenozoic history of the Lake Baikal depression and the formation of its unique biodiversity. Stratigraphy and Geological Correlation 19: 404-423. DOI: 10.1134/ S0869593811040058

Maximova N., Koroleva A., Sitnikova T. et al. 2017. Age dynamics of telomere length of Baikal gastropods is sex specific and multidirectional. Folia Biologica (Krakow) 65: 187-197. DOI: $10.3409 / \mathrm{fb} 65$-4.187

Salomons H.M., Mulder G.A., van de Zande L. et al. 2009. Telomere shortening and survival in free-living corvids. Proceedings. Biological Sciences 276: 3157-3165. DOI: 10.1098/rspb.2009.0517

Simon N., Smoller J., McNamara K. et al. 2006. Telomere shortening and mood disorders: preliminary support for a chronic stress model of accelerated aging. Biological Psychiatry 60: 432-435. DOI: 10.1016/j.biopsych.2006.02.004

Timoshkin O.A. 1994. Origin and evolution of the fauna of free-living Turbellaria of Lake Baikal. Zoologicheskiy Zhurnal [Zoological Journal] 73: 35-50. (in Russian)

Zhu Y., Liu X., Ding X. et al. 2019. Telomere and its role in the aging pathways: telomere shortening, cell senescence and mitochondria dysfunction. Biogerontology 20: 1-16. DOI: 10.1007/s10522-018-9769-1 